\title{
The medical workforce of the future
}

\author{
Doctors training now will have a very different world of practice. They will need to understand team \\ care, patient engagement in decision making and clinician leadership as well as the rapidly changing \\ health system. But what an exciting environment to be entering, with all the advances in care \\ available and expanding into the future - as long as we can afford them.
}

$\mathrm{T}$ his issue of MJA Open is about the role that doctors will play in the health system of the future, where and how they might work, how we might train them and how are we going to fund them.

The health and social welfare sector is now the largest workforce in Australia (1.4 million), not to mention the 2.3 million carers (both paid and unpaid) who provide support every day for those Australians who for many reasons cannot take care of themselves. This workforce is, like the rest of the population, ageing and wanting to work in different ways. What we can do for patients has changed so much over the past 20 years that it often bears little relation to what health professionals were taught at university - and this change is likely to continue into the future at an even more rapid pace. The importance of the health workforce is no better emphasised than by the Australian federal and state governments' commitment to establish Health Workforce Australia in 2009 with triennial funding of $\$ 1.4$ billion. This organisation has as its mission creating a more sustainable health workforce by 2025 (https://www.hwa.gov.au).

New knowledge and technology are great drivers of change in medicine, as in other disciplines. The increase in genetic testing in the past 10 years alone has been responsible for a major shift in diagnosis, treatment and prevention of many diseases. New imaging techniques have allowed diagnosis of conditions at a much earlier and often more easily treatable stage, such that diseases that killed 30 years ago (many forms of cancer, some metabolic disorders) are now curable, or patients can maintain a good quality of life with continuing therapy. There is little doubt it will be chronic diseases and dementia that will shape the challenge of delivering health services in the foreseeable future. ${ }^{1}$

These and other developments have prompted much discussion and review within the medical and broader health professions, in the community, and particularly within medical schools, where we are preparing young people for careers that will run for 40 or more years. One of the major challenges for educators and health professionals is accepting that health care is now delivered as a team activity by various health professionals; and one of the most important issues for health professional education is embedding those team care skills through various interprofessional learning experiences. ${ }^{2}$ That said, given the pivotal role that doctors play in health care, this MJA Open will concentrate on the role that doctors play in the health workforce.

The questions we face as we develop curricula, prepare exams, run tutorials and teach clinical skills are complex. It
Bruce G Robinson $M D, M S C$, FRACP Dean $^{1}$

Peter M Brooks MD, FRACP, FRCP(Glas), Director, Australian Health Workforce Institute $^{2}$

1 Sydney Medical School, University of Sydney, Sydney, NSW.

2 School of Population Health, University of Melbourne, Melbourne, VIC brooksp@ unimelb.edu.au

doi: 10.5694/mjaol2.10524 is easy enough to say that change will occur, but narrowing down the specifics of what and where - and preparing for such a future - provides a challenge of a different magnitude altogether. For example, how will we cope with clinical training demands from the increasing number of medical students, when patients in hospitals are now often unable to be as involved in teaching programs as they were a decade or two ago, because of the nature of their condition or the short period of their stay in hospital? What are the opportunities for clinical learning in private hospitals, in aged care and mental health facilities and in primary care? How can we effectively use the wonderful clinical experiences that abound outside the traditional tertiary teaching hospitals in primary care, aged care facilities, mental health services and in the community? All medical schools have embraced the need to increase clinical training opportunities to some extent through rural clinical school initiatives, by which students spend the bulk of their clinical training in the rural sector and achieve equivalent examination results to those trained in city hospitals. It should be acknowledged, however, that some schools have done better than others in this regard. ${ }^{3}$

So how different do we believe the practice of medicine will really be in the future? What tasks will doctors still need to do? What will patients want from us? How much of our daily routine will be performed by other health professionals? What role will IT (information technology) play in practice? Will consultations all take place remotely? Certainly, telemedicine is changing the way we deliver health services, but it has taken us over a decade from the emergence of data showing that it could work to reach the point where we actually have an item number allowing doctors to charge for this service.

More fundamentally, can we anticipate what changes are likely and prepare students accordingly? And how much of our focus should be on developing the skills to learn, to discover, to evaluate new knowledge as it becomes available and to understand change management? In this rapidly changing environment, perhaps we need to revisit our fairly recent focus on specialism and train more generalist health professionals who have greater flexibility to change career track as the environment around them changes.

\section{Much may not change}

A fundamental interaction between patient and doctor is the consultation or the procedure, and this may change little. Doctors have been taking histories and examining people for millennia. The nuances of communication and 
the time-honoured physical examination skills will still require personal contact between patient and provider, although significant amounts of this interaction may develop a "virtuality". The task of gathering and evaluating data through patient history-taking, physical examination and diagnostic testing to form a differential diagnosis, which is then tested and challenged until the diagnosis is confirmed, will not change. It will, however, be significantly enhanced by such things as an electronic health record owned by the patient and shared between professionals. This will surely rid us of much duplication of effort as patients move across the health system.

Some consultations may take place virtually, particularly among patients with chronic disease who need following up and people living in distant locations. It is also possible for people with diabetes to have their weight, exercise, blood glucose and $\mathrm{HbA}_{1 \mathrm{c}}$ all monitored via a mobile phone, and for communication with the doctor or other health professional to take place by phone or as a telehealth consultation. As health professionals, we often do not appreciate the time taken for both patients and professionals to travel between appointments. Yet, there is a need to quickly explore and evaluate such new models of health care delivery.

The attributes we look for in admitting students to our medical program - compassion, understanding, empathy, a sense of ethics - and the skills of problem solving, listening and communication will be critical in health professionals far into the future. ${ }^{4}$ Advances in biology and IT will never overshadow them.

Other aspects of medical practice, similarly, will change little over the next decades. Most of the sickest patients will continue to be treated in hospital, where doctors will increasingly be focused on resolving acute exacerbation of chronic health issues, treating life-threatening conditions such as cancer and managing severe trauma. Most of the medical profession's work will continue to occur in community settings, whether in general or specialist practice, but there will be a need to shift our focus to outof-hospital care models, as well as to get serious about disease prevention. ${ }^{5}$

Predictions even a decade ahead in a field such as medical workforce necessarily involve qualification. Having said that, we can be reasonably confident that the recent drivers of change - which have already altered the practice of medicine to varying degrees - will continue to be a factor into the foreseeable future. They will challenge experienced practitioners and young graduates alike. Issues such as the feminisation of the workforce, work-life balance, and safe hours clauses have had significant influences on training and on how we manage the workforce. Should we continue to rely on overseas-trained doctors, from countries who often need them much more than we do, to support our workforce - particularly in rural areas? The rapidly increasing costs of health care will also play a major role in stimulating new ways of approaching health care delivery. An uncapped fee-forservice system that encourages activity rather than rational care is unlikely to escape the eye of government funding circles for very much longer.
More than anything else, the way doctors operate in the future will be influenced by advances in IT, biology, pathology and therapy.

Every year, we will have new graphic insights into anatomical nooks and crannies and physiological or pathological functions in molecules that at the moment we may not even know exist. Tracking of metastatic cells, visualisation of oxygen-starved tissues and toxin-poisoned cells will be routine. Tumours will be phenotyped in far greater detail than at present. Patterns of expression of proteins in cancers and detection of minute quantities of these proteins in blood will change diagnosis, prognostication and our ability to tailor treatments to individual patients and their tumours. Drug sensitivities, toxicities and methods of administration will be refined. The therapeutic armamentarium will be expanded to include inhibitors and modifications of molecules yet to be identified. We may not yet have a complete understanding of the interaction between all the proteins expressed by our 25000 genes or between each of the two copies of our genes, but we will know our basic gene sequence. Advances in stem-cell therapies, bioengineering and nanotechnology will provide exciting opportunities both diagnostically and therapeutically.

But if advances in biology and improvements in pathology and imaging give us the potential for faster and more accurate diagnoses of diseases, doctors will be required to upgrade their skills or learn new skills.

More than ever before, they will need to be able to sort the wood from the trees. Collecting, critiquing, and interpreting the vast amounts of new information will be an increasingly important skill of future practitioners. Doctors will need high-order abilities in data management. Much of the information will be biological, so doctors will also need a thorough understanding of what is normal to recognise what is not so normal.

Research training, by which students and graduates become experienced in critical thinking and interpretation of new knowledge as it becomes available, will become increasingly important. There will be a need to encourage interdisciplinary research, encompassing engineering nanotechnology and IT, and to look for ways of appropriately funding this research. There is also a need for research on educational methods such as simulation, on health systems research and on how to implement new knowledge.

In all this, the roles of patient and doctor are likely to change. Doctors will not be the all-authoritative figure but part of, or the leader of, a team of professionals providing a range of care in an integrated, evidenced-based way. Leadership skills will be required by some and "followership" skills by all.

\section{The challenge of managing resources}

Managing health resources in an era of longer lives, high technology, increased expectations and rising incidence of chronic disease will be one of the key challenges for governments, communities and the medical profession.

In the decades ahead, resource allocation will need to change from acute care to preventive care. The changes 
will need to be supported by evidence, and will need to result in greater resources for prevention. Vaccinating young women and men against papilloma virus, for example, will save many lives and much morbidity and reduce the high cost of treating cervical cancer.

Resource allocation will be guided by engaged community discussions on subjects such as the need for advance health care directives, issues surrounding the right of access to high technology, high-cost end-of-life care, allocation of resources to people with disability, consumers' responsibility for self-care, and roles and responsibilities of families in providing care. Ideally, resource allocation will be without political or professional self-interest, and will balance the best interests of the individual against those of the population.

Doctors will need to be fully involved in critical decisions on resource allocation, but to fully participate they will need to be informed by research and able to take responsibility for the active enquiry which informs policies and decisions.

Doctors should be the key advocates for our health system and our patients. Hopefully, by 2025, we will have long moved on from the current cost-shifting between state and federal governments; but the risk of bureaucratic armies or ill informed decisions that result in funds being diverted to meet political or other objectives rather than spent on health care will always be there.

\section{Changing populations and diseases}

It is almost certain that patients, even a decade ahead, will be different. Immigration patterns are already changing Australia's population, and medicine is increasingly a profession where people have the opportunity to (and do) work in all corners of the globe.

Disease patterns will likely be different. If the global epidemic of obesity is unchecked, people with diabetes, blindness, renal failure, stroke, myocardial and peripheral ischaemia and arthritis will overwhelm our hospitals. Infectious diseases will change if global warming continues; dengue and many other arthropod-borne diseases will occur in temperate climates. People will be more mobile, and diseases seen infrequently in Australia will likely become more prevalent.

Doctors of the future will need to have a broad understanding of the scope of medical practice in Australia and internationally. Their training and experience will have given them experience in the public and private health systems, and in metropolitan and rural and remote Australia.

Understanding of the needs of patients in these different settings and areas will enable better allocation of resources and the provision of better care. This is more a wish than a prediction, but we believe that if all doctors spend a period of time - either during medical school training or after in a resource-poor health care setting in a developing country, it would provide them with insight and experience of advanced pathology and the scale of international suffering and health inequity.

\section{The future?}

As educators, our role is to try to anticipate these changes and prepare our graduates with the skills to drive change and thrive on uncertainty. These skills include not only research training and lifelong learning, but the management and leadership skills to predict and forge the changes that will inevitably occur in their own practising lives.

For this MJA Open, we have asked authors to be provocative and challenge the status quo and to project their area of expertise into the future and see how the medical workforce will sit within the health system in 2025. This must be from the perspective of how we fund health care, who will be working in it, international migration of health workers, and how we get doctors to work in rural areas and with disadvantaged populations. We are sure there are areas we have not covered, but we hope this publication will stimulate your contributions to medical workforce solutions.

Competing interests: No relevant disclosures

Provenance: Commissioned; externally peer reviewed.

1 Gorman DF, Brooks PM. On solutions to the shortage of doctors in Australia and New Zealand. Med J Aust 2009; 190: 152-156.

2 Pozner S, Hylin U, Kusoffsky A, et al. Interprofessional training in the context of clinical practice: goals and students' perceptions on clinical education wards. Med Educ 2004; 38: 727-736.

3 Couper ID, Worley PS. Meeting the challenges of training more medical students: lessons from Flinders University's distributed medical education program. Med J Aust 2010; 193: 34-36.

4 Oates K, Goulston K. How to select the doctors of the future. Intern Med J 2012; 42: 364-369.

5 Barker R. 2030: the future of medicine - avoiding medical meltdown. New York: Oxford University Press, 2011. 